Ausgehend von den weltweiten Ungleichgewichten in der Weltschiffahrt und im Welthandel (Anteil der Dritten Welt an der Welthandelsflotte $6 \%$ ) behandelt Yeats die Folgen für die Dritte Welt, wobei im Mittelpunkt die langfristigen Entwicklungen der Transportkosten (auch im historischen Vergleich mit der säkularen Rolle von Schiffahrt und Transportkosten in der industriellen Entwicklung entwickelter Länder) und die institutionellen Rahmenbedingungen, bzw. Restriktionen für die Entwicklungsländer stehen. Die Analyse wird durch theoretische Erörterungen, Effekte von Transportkostensteigerungen auf den Handel, ergänzt.

Yeats' Versuch, diese für die Dritte Welt eminent wichtigen Probleme theoretisch und ökonomisch-politisch zu klären, muß allerdings als gescheitert angesehen werden. Zwar sind seine Erhebungen zu den Entwicklungen der Transportkosten sehr bedeutsam, aber gerade die Intentionen der Dritten Welt, die institutionellen Rahmenbedingungen im internationalen Seeverkehr durch eine kollektive Strategie (bspw. Neuordnung des internationalen Linienverkehrs durch den Verhaltenskodex für Linienkonferenzen, Transformation der offenen in normale Register und das "bulk sharing" in der Massengutschiffahrt) zu beseitigen, erfaßt Yeats nur sehr oberflächlich. Dies muß umso mehr erstaunen als Yeats als "First Economics Officer" der UNCTAD, die die Bausteine der Neuen Internationalen Seeverkehrsordnung entwickelt hat, sehr guten Einblick in den derzeitigen Stand der Schiffahrtsentwicklung der Dritten Welt hat. Zweifelsohne stellen Transportkosten und institutionelle Bedingungen im Weltseeverkehr (bspw. monopolartige Macht der Industrieländer) wesentliche Barrieren für die Schiffahrt der Entwicklungsländer dar, aber sicherlich nicht die einzigen, was sich u. a. an den Schwierigkeiten von Dritte Welt-Reedereien, genügend Kapital zum Schiffserwerb aufzubringen, an mangelndem technischem know-how, an fehlendem Management und Seeleuten und den politisch-institutionellen Bedingungen in der Dritten Welt, zeigen läßt.

Trotz dieser Einwände stellen Yeats' Aussagen zum Problem der Transportkosten einen wesentlichen, auch theoretischen, Beitrag zu den ökonomischen Restriktionen im internationalen Seeverkehr dar, allerdings keine "integrierte Einschätzung von Schiffahrt und Entwicklungspolitik", wie der Titel des Buches verspricht.

Robert Kappel

\title{
Lloyd Rodwin
}

\section{Cities and City Planning}

Plenum Publ., New York and London 1981, \$29.50

Weltweit befindet sich die Stadt in einer Krise. In den industrialisierten Staaten ist dies eine Krise der Kernstädte, die sich in Bevölkerungs-, Funktions- und Einkommensverlusten manifestiert, und zu einem allmählichen Verfall der Kernstädte führt. Demgegenüber wird die Krise der Stadt in den Entwicklungsländern in erster Linie durch ein 
hypertrophes und unkontrolliertes Wachstum hervorgerufen, mit der Folge fehlender Erwerbsmöglichkeiten, unzureichender kommunaler Dienstleistungen, sich rasch ausbreitender Elendsviertel und einer notorischen Mittelknappheit. Mit dieser Realität sieht sich der Stadt- und Regionalplaner der Gegenwart nach Auffassung von Lloyd Rodwin, selbst ein prominenter Vertreter dieser Disziplin, konfrontiert.

Rodwins neues Buch "Cities and City Planning" - eine Sammlung von 14 Aufsätzen, die in fünf Hauptteilen präsentiert werden, ist in erster Linie eine Bestandsaufnahme der wissenschaftlichen Diskussion im Fach Stadt- und Regionalplanung. Hauptgegenstand der Untersuchungen ist die Frage nach den unterschiedlichen und sich im Laufe der Zeit wandelnden Ansätze, Methoden, Erwartungen und Ziele der Stadt- und Regionalplaner, die diese in den letzten 25 Jahren den krisenhaften Entwicklungen der Stadt entgegenzusetzen suchten.

Teil I des Buches beschäftigt sich vornehmlich mit Theorie- und Perzeptionsproblemen der Stadt. Im Zentrum dieser Erörterungen stehen Typlogisierungsversuche sowie Uberlegungen zum Stellenwert und Erscheinungsbild der Stadt aus der Sicht unterschiedlichster Beobachtergruppen: Laien, Wissenschaftler, Politiker und Planer.

Im zweiten Hauptteil analysiert Rodwin Probleme der Kommunen in industrialisierten Staaten. Besondere Aufmerksamkeit widmet er dabei den planerischen Bemühungen zur Bewältigung dieser Probleme. Ein ebenso spektakulärer wie kühner Versuch dazu, der auf viele Stadt- und Regionalplaner eine geradezu magische Anziehungskraft ausübte, war das "New Communities" oder "New Town" Program, mit dem man in den vergangenen Dekaden zuerst in Großbritannien und später dann auch in den USA die kommunalen Probleme angehen wollte. Doch finanzielle Fehlplanungen, mangelnde politische Unterstützung, ungünstige ökonomische Rahmenbedingungen, administrative Koordinationsprobleme, eine verfehlte Standortwahl und die Ausklammerung der ärmeren Einkommensgruppen aus den Projekten ließen das amerikanische "New Communities Program" letztlich kläglich scheitern.

Teil drei setzt sich mit der Stadt- und Regionalplanung in Entwicklungsländern auseinander. Ganz generell stellt Rodwin dabei eine durchaus positive Entwicklung des Faches fest, die sich im Wandel von einer rein technokratisch-wachstumsorientierten zu einer stärker von sozio-politischen UUberlegungen geleiteten Planungskonzeption zeigt. So hätten mittlerweile Begriffe wie Partizipation und Dezentralisierung, aber auch eine intensive Beschäftigung mit den Problemen des informellen Sektors und Forderungen nach Abbau der eklatanten horizontalen und vertikalen Ungleichheiten Eingang in das planerische Denken gefunden.

Diese positiven Entwicklungen haben jedoch nach Auffassung Rodwins den wissenschaftlichen Stand des Faches in den Entwicklungsländern nur wenig beeinflussen können. Rodwin konstatiert hier vor allem große Ausbildungsdefizite: nicht nur gebe es viel zu wenig gut ausgebildete Planer, sondern es liege der Ausbildungsschwerpunkt noch immer ausschließlich auf den traditionellen Bereichen der physischen und Flächennutzungsplanung. Desweiteren kritisiert Rodwin, daß sich die meisten Planer weitgehend an 
Stadtentwicklungskonzepten orientieren, die möglicherweise für die Städte in den entwickelteren Staaten angemessen seien, nicht jedoch für die Städte der Entwicklungsländer, was zwangsläufig schwere Fehlplanungen nach sich ziehen müsse. Es sei daher auch wenig verwunderlich, wenn diese Planer gerade für die Armutsprobleme ihrer Heimatländer nur wenig Verständnis aufbrächten.

Im vorletzten Teil stehen vornehmlich Ausbildungsprobleme zur Diskussion - vor allem mit Blickwinkel auf die im vorangehenden Kapitel diagnostizierten Defizite in der Dritten Welt. Um gerade hier bessere Planungsresultate zu erzielen, fordert Rodwin eine zweijährige praxisorientierte Planerausbildung, die bereits im undergraduate-Stadium erfolgen soll mit einer verstärkten Einbeziehung sozialwissenschaftlicher Fächer in die Curricula.

Im letzten Teil des Buches endlich reflektiert der Autor die Diskrepanzen zwischen Illusionen und Realitäten des Planerberufs. Er warnt davor, in der Planung das Allheilmittel zur Korrektur gesellschaftlicher Fehlentwicklungen zu sehen: die aktuellen Einflußmöglichkeiten und die Beiträge des Planers zur Lösung anstehender Probleme würden häufig weit überschätzt.

Diese Inhaltsangabe bereits läßt erkennen, daß der Titel des Buches etwas irreführend ist. Wer die Diskussion gegenwärtiger Stadtentwicklungs- und Stadtplanungsprobleme etwa am Beispiel ausgewählter Städte - erwartet, wird sicher enttäuscht sein. Dagegen behandelt Rodwin vornehmlich wissenssoziologische und theoretische Fragestellungen seines Faches. Die Stärken dieser Aufsatzsammlung liegen dabei zweifellos in ihrer kritischen Bewertung der Leistungen und Problemlösungskapazitäten der Disziplin insbesondere auch, was die Bewältigung der drängenden Verstädterungsprobleme in der Dritten Welt angeht. Hervorzuheben ist dabei der breite wissenschaftliche Horizont des Autors, der ihm beispielsweise erlaubt, die Möglichkeiten und Grenzen der Sozialwissenschaften für den Planer angemessen zu bewerten. Bemerkenswert ist auch das Abrücken Rodwins von einem rein technokratischen Planungsverständnis, wie es noch in den fünf ziger und sechziger Jahren die Disziplin beherrschte. Stadt- und Regionalplanung heißt für Rodwin nicht allein physische oder Flächennutzungsplanung, sondern schließt auch die nationale Dimension der Urbanisierung (Forderung einer nationalen Urbanisierungsstrategie) und das soziale und politische Umfeld ein.

Während der Aufsatz "The Form of the City" interessante theoretische Uberlegungen enthält, bereichern die an anderer Stelle angestellten Typplogisierungsversuche des Autors die bereits vorhandenen Typologien der Stadt nur um eine weitere Variante, ohne dabei aber allzuviel analytischen Erkenntnisgewinn zu erbringen. Zweifellos ist die Zusammenfassung und Klassifizierung der Meinungen, Schulen und Ansätze einer Disziplin ein wichtiges und verdienstvolles Unterfangen, doch kommt man dabei nicht an der Feststellung vorbei, daß Rodwins Buch im Grunde auf eigene neue Ansätze verzichtet.

Rodwins Buch ist eine anspruchsvolle Lektüre vor allem für diejenigen Stadt- und Regionalplaner, die ihre praktische Tätigkeit und ihren wissenschaftlichen Standort 
einer stetigen kritischen Reflektion unterziehen. Darüber hinaus ist es empfehlenswert für jeden Leser, der an der Geschichte und Entwicklung der Disziplin Stadt- und Regionalplanung Interesse findet.

Jürgen Rüland

\section{Thomas Buergenthal/Robert Norris/Dinah Shelton}

\section{Protecting Human Rights in the Americas. Selected Problems}

N. P. Engel Verlag, Kehl, Straßburg, Arlington, 1982, XVII, 337 S., DM 48,-

Der hier anzuzeigende Band ist ein außerordentlich gelungenes Arbeitsbuch, das erstmals in umfassender Weise Material zum Verständnis des neuen völkerrechtlichen Menschenrechtsschutzsystems im amerikanischen Doppelkontinent bereitstellt. Seit dem Inkrafttreten der Amerikanischen Menschenrechtskonvention im Jahre 1978 und der Errichtung des Inter-Amerikanischen Gerichtshofs für Menschenrechte besteht neben dem europäischen System eine zweite regionale Menschenrechtsordnung. Die Mitgliedsstaaten der OAS haben deren normative Ausgestaltung stark an das europäische Vorbild angelehnt, sind nicht den Weg der OAU gegangen, die ihrer Menschenrechtskonvention von 1981 (die noch nicht in Kraft getreten ist) zahlreiche eigene Charakteristika gegeben, insbesondere auf die Schaffung eines Menschenrechts-Gerichtes verzichtet hat. Auf das künftige Schicksal beider Modelle darf man gespannt sein. Das vorliegende Textbuch enthält Auszüge aus dem wissenschaftlichen Schrifttum, Dokumente aus der Arbeit der Amerikanischen Menschenrechtskommission und formuliert Fragen, die nicht nur den Völkerrechtsunterricht anregen, sondern auch dem Wissenschaftler Stoff zum Nachdenken geben können. Die Zusammenstellung zeugt von souveräner Kenntnis der Praxis, ohne die dogmatischen Fragen geringzuschätzen. Insbesondere die weitere Erforschung der Befugnisse der Kommission (deren Engagement 1980 schon zu einer Krise der OAS geführt hat) und die Bewertung ihrer bisherigen Rolle unter dem Gesichtspunkt der Effektivität werden durch den Band wesentlich erleichtert. Es ist zu wünschen, daß die Autoren ihn fortschreiben mögen, sobald der Interamerikanische Gerichtshof für Menschenrechte seine ersten Sachentscheidungen gefällt haben wird.

Philip Kunig 